\title{
Grape seed proanthocyanidin extract alleviates urethral dysfunction in diabetic rats through modulating the NO-cGMP pathway
}

\author{
BING ZHANG ${ }^{1,2 *}$ ZHAOCUN ZHANG ${ }^{2 *}, \mathrm{HONG} \mathrm{JI}^{3}, \mathrm{HUI} \mathrm{SHI}^{4}, \mathrm{SHOUZHEN} \mathrm{CHEN}^{2}$, \\ DONGLIANG YAN ${ }^{1}$, XUEWEN JIANG ${ }^{2}$ and BENKANG SHI ${ }^{2}$ \\ ${ }^{1}$ Department of Urology, Binzhou Medical University Hospital, Binzhou, Shandong 256600; \\ ${ }^{2}$ Department of Urology, Qilu Hospital, Shandong University, Jinan, Shandong 250012; Departments of ${ }^{3}$ Pathology and \\ ${ }^{4}$ Bone and Joint Surgery, Binzhou Medical University Hospital, Binzhou, Shandong 256600, P.R. China
}

Received August 13, 2015; Accepted September 9, 2017

DOI: $10.3892 /$ etm.2017.5499

\begin{abstract}
Oxidative stress is closely associated with the onset of diabetes mellitus (DM). Diabetic urethropathy is one of the most common complications of DM, but few studies have been conducted to investigate the role of oxidative stress in diabetic urethropathy. Grape seed proanthocyanidin extract (GSPE) has been previously reported to reduce oxidative injury. The present study aimed to investigate the role of oxidative stress and the protective effects of GSPE on urethral dysfunction using a streptozotocin-induced DM rat model. Female Wistar rats were divided into a control group $(\mathrm{n}=36)$, a DM group $(\mathrm{n}=36)$ and a DM + GSPE group $(\mathrm{n}=36)$. Urodynamic testing was performed using a PowerLab data acquisition device. The expression of neuronal nitric oxide synthase (nNOS), 3-nitrotyrosine and nuclear factor erythroid 2-related factor 2 (Nrf2) was determined using western blot analysis. The expression of 3-nitrotyrosine was also determined using immunohistochemistry. Nitric oxide (NO), cyclic guanosine monophosphate (cGMP), superoxide dismutase (SOD), glutathione peroxidase (GSH-Px) and malondialdehyde (MDA) were measured using commercial ELISA kits. A significant increase was observed in the intravesical pressure thresholds for inducing urethral relaxation and the urethral perfusion pressure nadir in DM rats compared with the control group. GSPE was observed to reverse the increase of these parameters compared with the DM group. In addition, GSPE could reverse
\end{abstract}

Correspondence to: Professor Benkang Shi, Department of Urology, Qilu Hospital, Shandong University, 107 Wenhua West Road, Jinan, Shandong 250012, P.R. China

E-mail: shibenkang@sina.com

*Contributed equally

Key words: diabetes mellitus, urethra, oxidative stress, grape seed proanthocyanidin, nitric oxide-cyclic guanosine monophosphate pathway the downregulation of nNOS, NO and cGMP expression, and the decreased activities of antioxidant enzymes (SOD and GSH-Px). GSPE reversed the upregulation of 3-nitrotyrosine and MDA in DM rats. GSPE also activated Nrf2, which is a key antioxidative transcription factor. The findings of the present study demonstrated that GSPE protects urethra function in DM rats through modulating the NO-cGMP signaling pathway. The protective roles of GSPE may be associated with activation of the Nrf2 defense pathway.

\section{Introduction}

Diabetes mellitus (DM) refers to a group of metabolic disorders characterized by hyperglycemia, which have a severe impact on public health. To date, the majority of studies examining DM complications have focused on the pathogenesis and management of diabetic cystopathy, one of the most common complications of DM $(1,2)$. Diabetic cystopathy is considered to be a manifestation of polyneuropathy, involving sensory and autonomic nerve fibers 1 (3). A previous study reported that DM induces damage to the innervation of the urethra, together with reduction of urethral relaxation during micturition reflex (4). DM may also induce impairment of urethral smooth muscle, which is closely associated with urethral dysfunction. However, few studies have been conducted to investigate the mechanism of urethral dysfunction in DM, so this remains unclear.

Precise coordination between smooth muscle in the urethra and bladder is crucial for the balance between urine storage and bladder emptying (4). Such coordination is largely dependent on the nitric oxide (NO) released from nerve endings of parasympathetic nerves, which serves as a major neurotransmitter $(5,6)$. NO can diffuse into the smooth musculature of the urethra, in which it stimulates the synthesis of cyclic guanosine monophosphate (cGMP) and finally induces smooth muscle cell relaxation (7).

Previous studies $(8,9)$ have suggested that diabetic patients are under a state of oxidative stress characterized by a relative overload of oxidants. In these patients, free oxygen radicals, especially superoxide $\left(\mathrm{O}_{2}^{-}\right)$, are aberrantly accumulated in vivo. 
The increased $\mathrm{O}_{2}{ }^{-}$could reduce the bioavailability of $\mathrm{NO}$ via binding with NO rapidly to form peroxynitrite (ONOO) (10). Thus, it seems reasonable to assume that urethral dysfunction during reflex micturition in DM would be associated with the decreased bioavailability of $\mathrm{NO}$ and/or the over-production of ONOO-

Grape seed proanthocyanidin extract (GSPE), a natural polyphenolic compound extracted from grape seeds, has exhibited a broad spectrum of pharmacological and therapeutic properties against oxidative stress (11). Previously, studies have been performed to investigate the protective effects of GSPE in various disorders induced by oxidative stress, such as diabetic nephropathy (12), obesity (13) and cardiac disorders (14). For the protective effects of GSPE in rats with diabetic nephropathy, the authors concluded that GSPE could ameliorate the diabetic nephropathy through a reduction in oxidative stress and an increase in renal antioxidant enzyme (12). Nuclear factor erythroid 2-related factor 2 (Nrf2) is involved in regulating the cellular antioxidative responses and redox status. In addition, previous results have suggested that activation of the Nrf2 pathway serves a critical function in myocyte differentiation as well as muscular contractile and metabolic properties in a diabetic model of muscle atrophy (15). The present study aimed to investigate the antioxidant effects of GSPE in the pathogenesis of urethral dysfunction in DM, in order to evaluate a potential mechanism of its protection against urethral dysfunction. The precise protective effects of GSPE were also investigated.

\section{Materials and methods}

Animals and drugs. A total of 108 female Wistar rats (8-weeks-old, weighing $257.7 \pm 18.21 \mathrm{~g}$ ) were purchased from the Animal Center of Shandong University (license number: SCXX20090010; Jinan, China). The rats were fed under a $12 \mathrm{~h} \mathrm{light/dark} \mathrm{cycle} \mathrm{and} \mathrm{had} \mathrm{free} \mathrm{access} \mathrm{to} \mathrm{standard} \mathrm{rat} \mathrm{chow}$ and tap water in an environment at $24^{\circ} \mathrm{C}$ with $60 \%$ humidity. The rats were randomly divided into three groups (Fig. 1): i) control group ( $\mathrm{n}=36)$, administered with $0.1 \mathrm{M}$ citrate buffer (pH 4.5, $4 \mathrm{ml} / \mathrm{kgc}$, at no. C-0071; Shanghai Enzyme-linked Biotechnology, Shanghai, China) via intraperitoneal injection, followed by intragastric injection of $0.9 \%$ normal saline $(4 \mathrm{ml} / \mathrm{kg})$ once a day for 8 weeks; ii) DM group $(\mathrm{n}=36)$, administered with a single dose of $65 \mathrm{mg} / \mathrm{kg}$ streptozotocin (STZ; Sigma-Aldrich; Merck KGaA, Darmstadt, Germany) dissolved in $0.1 \mathrm{M}$ citrate buffer ( $\mathrm{pH} 4.5$ ) via intraperitoneal injection, followed by intragastric injection of $0.9 \%$ normal saline $(4 \mathrm{ml} / \mathrm{kg})$ once a day for 8 weeks; and iii) DM+GSPE group $(n=36)$, which were administered with a single dose of $65 \mathrm{mg} / \mathrm{kg}$ STZ via intraperitoneal injection followed by intragastric injection of GSPE extracted from European red grape seed (Cabernet sauvignon) once a day for 8 weeks ( $250 \mathrm{mg} / \mathrm{kg}$, proanthocyanidin content $>96 \%$; Tianjin Jianfeng Natural Product R\&D Co., Ltd., Tianjin, China). Subsequently, 10 rats selected randomly from each group (10/36) were placed in metabolic cages to measure body weight, blood glucose and urine volume. All experimental procedures were compliant with the Guidelines for Laboratory Animal Use and Care from the Chinese Center For Disease Control and Prevention and the Rules for Medical Laboratory Animals from the Chinese
Ministry of Health, under protocol CAU-AEC-2013-073 (16) and were approved by the Ethical Committee of Qilu Hospital of Shandong University (Jinan, China; permit no. DWLL-20B-025). All surgeries (urodynamic testing and isolation of urethra) were performed under pentobarbital anesthesia (intraperitoneal), and rats were sacrificed after sample collection by pentobarbital anesthesia (intraperitoneal) followed by cervical dislocation. All efforts were made to minimize suffering.

Simultaneous recordings of urethral perfusion pressure (UPP) and intravesical pressure under isovolumetric conditions. Simultaneous recordings of UPP and intravesical pressure were monitored as previously described (17). Briefly, the rats ( $n=6$ per group) were anesthetized with $35 \mathrm{mg} / \mathrm{kg}$ pentobarbital via intraperitoneal injection. Then, a midline abdominal incision was made to expose the bladder and proximal urethra. The two ureters were tied distally and cut to prevent urine filling in the bladder. Subsequently, a bladder catheter and double lumen urethral catheter were inserted through the bladder dome by separate cystostomy. Upon fixing in the bladder neck, the double lumen urethral catheter consisted of an outer cannula of PE-160 $(160 \mu \mathrm{m})$ and an inner cannula of PE-50 (50 $\mu \mathrm{m})$ for infusing saline and recording UPP, respectively. For the infusion of normal saline, the PE-160 cannula was connected to a pump and the velocity of infusion was set at $0.075 \mathrm{ml}$ per minute, in order to induce rhythmic isovolumetric contractions. PowerLab V30 (Laborie Medical Technologies Inc., Beijing, China) was used to determine the intravesical pressure under isovolumetric conditions, UPP nadir, baseline UPP and intravesical pressure thresholds for inducing urethral relaxation in each group.

Urethra tissue collection. Urethra tissue was collected and transferred to an animal operating table. A $1.0 \mathrm{~cm}$ epidural sheath was catheterized into the urethra as a support shaft to mark the urethra. Afterwards, a midline abdominal incision was made to expose the urethra. The urethra was distinguished along the sheath and was removed for western blotting and immunohistochemistry. The isolated tissues were stored at $-80^{\circ} \mathrm{C}$ until analysis.

Western blot analysis. The expression of neural nitric oxide synthase (nNOS), 3-nitrotyrosine and Nrf2 was determined using western blotting. Urethra tissues were homogenized in lysis buffer (P0013B, Beyotime Institute of Biotechnology, Haimen, China) containing protease inhibitors. Protein concentration was determined using commercial BCA kit (Beyotime Institute of Biotechnology). Protein $(40 \mu \mathrm{g})$ was separated on $10 \%$ SDS-PAGE gel and transferred to a $0.22 \mu \mathrm{m}$ polyvinylidene difluoride membrane. The membranes were blocked with $5 \%$ fat-free milk at $25^{\circ} \mathrm{C}$ for $1 \mathrm{~h}$ and incubated with primary antibodies against nNOS (1:1,000; C12H1; Cell Signaling Technology, Inc., Danvers, MA, USA), 3-nitrotyrosine (1:2,000; ab61392; Abcam, Cambridge, UK) and Nrf2 (1:2,000; ab31163; Abcam) overnight at $4^{\circ} \mathrm{C}$, and then incubated with goat anti-rabbit secondary antibody $(1: 4,000$, cat no. sc2004; Santa Cruz Biotechnology, Inc., Dallas, TX, USA) at $25^{\circ} \mathrm{C}$ for $1 \mathrm{~h}$. The same membranes were probed with an antibody against 


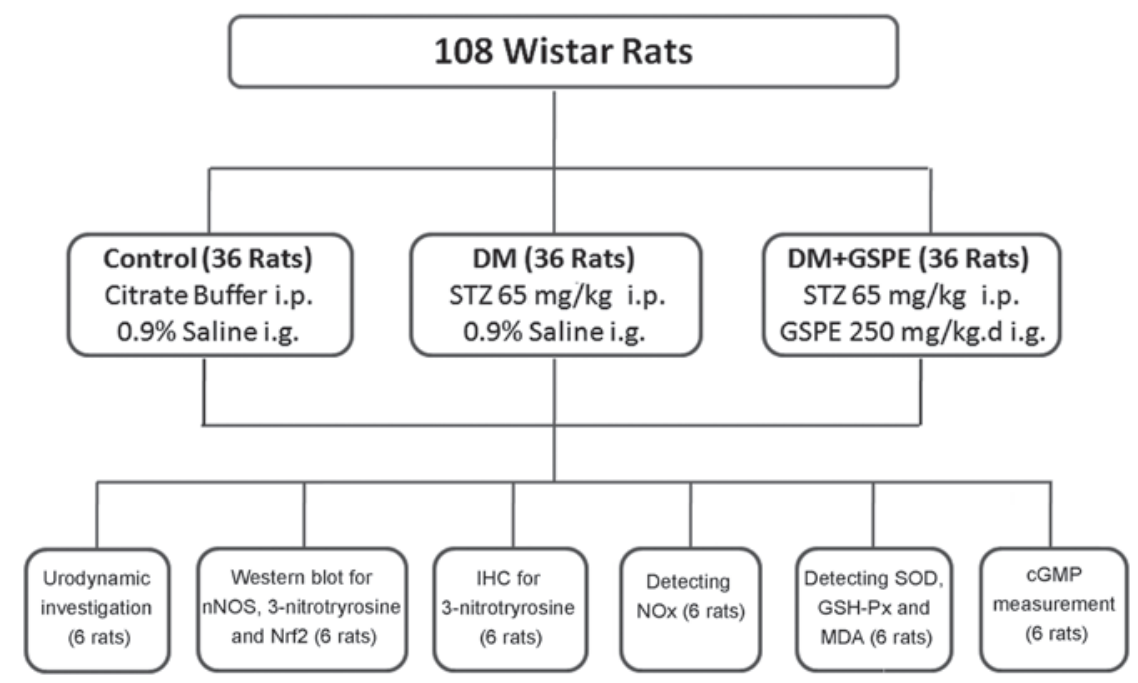

Figure 1. Animal grouping and methods of analysis. i.p., intraperitoneal injection; i.g., intragastric administration; IHC, immunohistochemistry; STZ, streptozotocin; GSPE, grape seed proanthocyanidin extract; nNOS, neural nitric oxide synthase; $\mathrm{Nrf2}$, nuclear factor erythroid 2-related factor 2; $\mathrm{NO}_{\mathrm{x}}$, total nitrate/nitrite; SOD, superoxide dismutase; GSH-Px, glutathione peroxidase; MDA, malondialdehyde; cGMP, cyclic guanosine monophosphate.

$\beta$-tubulin (1:400; cat no. AT809; Beyotime Institute of Biotechnology) for loading control. The results were visualized using an ECL chemiluminescence kit (cat no. P0018; Beyotime Institute of Biotechnology) Finally, densitometric values were quantified using a Kodak Image Station 2000 MM (Kodak, Rochester, NY, USA).

Immunohistochemistry. Urethra tissue sections $(3 \mu \mathrm{m})$ were fixed using $4 \%$ paraformaldehyde for $24 \mathrm{~h}$ and embedded in paraffin at room temperature. Upon dewaxing, the paraffin sections were immersed into EDTA antigen retrieval solution (cat no. P0085; Beyotime Institute of Biotechnology) at $92-98^{\circ} \mathrm{C}$ for $20 \mathrm{~min}$, followed by $0.3 \%$ hydrogen peroxide for $10 \mathrm{~min}$ to block endogenous peroxidase. Afterwards, the sections were blocked using $5 \%$ bovine serum albumin (cat no. ST023; Beyotime Institute of Biotechnology) for $30 \mathrm{~min}$ at $37^{\circ} \mathrm{C}$, and incubated with rabbit monoclonal antibody against 3-nitrotyrosine (1:200; cat no. ab61392; Abcam) for $18 \mathrm{~h}$ at $4^{\circ} \mathrm{C}$. After washing with PBS, the sections were incubated with peroxidase-conjugated secondary antibodies (1:200, cat no. sc-2345; Santa Cruz Biotechnology, Inc.) at room temperature for $1 \mathrm{~h}$. Finally, the sections were stained using diaminobenzidine at room temperature for $30 \mathrm{sec}$. Positive staining was visualized as a brown reaction product in the cytoplasm. Histological analysis was performed using a light microscope with a computerized imaging system (Northern Eclipse 7.0; Empix Imaging, Inc., Mississauga, ON, Canada) by two qualified observers blinded to the study. The degree of staining was evaluated based on the intensity score, as previously described (18), in which a score of 0-3 represented absence of staining, weak, moderate and strong staining, respectively. The proportional score was the percentage of cells that stained positive in different cell groups and was scored as: 0 , no positivity; $1,<1-25 \% ; 2,26-50 \% ; 3,51-75 \%$; 4 , $76-100 \%$ positive staining.

Estimation of oxidative stress in the urethra. Oxidative stress in the urethra was measured as described previously (18).
Glutathione peroxidase (GSH-Px, cat no. S1058; Beyotime Institute of Biotechnology), superoxide dismutase (SOD, cat no. S0109; Beyotime Institute of Biotechnology) and malondialdehyde (MDA, cat no. S1031, Beyotime Institute of Biotechnology) assays in urethra tissues were conducted using ELISA kits and evaluated by spectrophotometry, according to the manufacturer's protocol (Beyotime Institute of Biotechnology). GSH-Px activity was measured using the enzyme-catalyzed reaction product (reduced glutathione) and the absorbance was recorded at a wavelength of $412 \mathrm{~nm}$. The measurement of SOD activity was based on the combination of xanthine and xanthine oxidase at a wavelength of $550 \mathrm{~nm}$. The MDA level was detected using the thiobarbituric acid method with a maximum absorbance at $532 \mathrm{~nm}$ (19).

Determination of nitrate/nitrite. Total nitrate/nitrite $\left(\mathrm{NO}_{x}\right)$ levels, as the final metabolites of NO, were measured in urethra samples using an $\mathrm{NO}_{x}$ colorimetric assay kit (cat no. S0023; Beyotime Insititute of Biotechnology), according to the manufacturer's protocol. The $\mathrm{NO}_{\mathrm{x}}$ production was determined with the use of a Scientific Multiskan MK3 spectrophotometer (Thermo Fisher Scientific, Inc., Waltham, MA, USA) at $540 \mathrm{~nm}$.

Measurement of urethral cGMP content. Urethra tissues obtained from rats were used for the urethral cGMP content determination. The concentration of cGMP was evaluated using a cGMP-EIA kit purchased from Cayman Chemical Company (cat no. 581021; Ann Arbor, MI, USA), according to the manufacturer's protocol. The yellow-colored product formed with the EIA is inversely proportional to the amount of cyclic nucleotide present in the sample and was detected at $412 \mathrm{~nm}$ by a plate reader (Victor2 1420; PerkinElmer, Inc., Waltham, MA, USA).

Statistical analysis. Data are presented as the mean \pm standard deviation. Experiments were performed at least in triplicate. Data were analyzed using SPSS 19.0 (IBM Corp., Armonk, 
Table I. Body weight, blood glucose and urine volume.

\begin{tabular}{llcr}
\hline Group & Body weight $(\mathrm{g})$ & Blood glucose $(\mathrm{mg} / \mathrm{dl})$ & 24-h urine volume $(\mathrm{ml})$ \\
\hline Control $(\mathrm{n}=10)$ & $310.61 \pm 9.78$ & $114.2 \pm 13.4$ & $11.69 \pm 1.20$ \\
DM group $(\mathrm{n}=10)$ & $250.47 \pm 17.91^{\mathrm{a}}$ & $449.6 \pm 57.9^{\mathrm{a}}$ & $44.47 \pm 4.29^{\mathrm{a}}$ \\
DM+GSPE group $(\mathrm{n}=10)$ & $254.42 \pm 19.32^{\mathrm{a}}$ & $443.3 \pm 47.0^{\mathrm{a}}$ & $42.74 \pm 4.03^{\mathrm{a}}$ \\
\hline
\end{tabular}

${ }^{\mathrm{a}} \mathrm{P}<0.01$ vs. control group. DM, diabetes mellitus; GSPE, GSPE, grape seed proanthocyanidin extract.

Table II. UPP and intravesical pressure.

\begin{tabular}{lcc} 
Group & UPP nadir $\left(\mathrm{cm} \mathrm{H}_{2} \mathrm{O}\right)$ & inducing urethral relaxation $\left(\mathrm{cm} \mathrm{H}_{2} \mathrm{O}\right)$ \\
\hline Control $(\mathrm{n}=6)$ & $22.37 \pm 1.93^{\mathrm{a}}$ & $14.44 \pm 1.18^{\mathrm{a}}$ \\
DM group $(\mathrm{n}=6)$ & $36.24 \pm 2.66$ & $24.24 \pm 1.15$ \\
DM+GSPE group $(\mathrm{n}=6)$ & $24.99 \pm 2.05^{\mathrm{a}}$ & $17.86 \pm 4.74^{\mathrm{a}}$ \\
\hline
\end{tabular}

${ }^{\mathrm{a}} \mathrm{P}<0.01$ vs. DM group. DM, diabetes mellitus; GSPE, grape seed proanthocyanidin extract; UPP, urethral perfusion pressure.

NY, USA). One-way analysis of variance followed by a Tukey's post hoc test was used for inter-group comparisons. $\mathrm{P}<0.05$ was considered to indicate a statistically significant difference.

\section{Results}

GSPE exerts no obvious effects on body weight, blood glucose or urine volume. A significant decrease in body weight was observed in the DM group compared with the control $(\mathrm{P}<0.05$; Table I). However, no significant difference was observed in the DM + GSPE group compared with the DM group. The concentration of blood glucose was significantly increased in the DM group compared with the control group $(\mathrm{P}<0.05$; Table I), but no significant difference in blood glucose was observed in the DM + GSPE compared with the DM group. The urine volume was significantly increased in the DM group compared with the control group $(\mathrm{P}<0.05$; Table I), but no significant difference in urine volume was observed in the $\mathrm{DM}+$ GSPE compared with the DM group.

GSPE ameliorates DM-induced dysfunction in urethral relaxation. Baseline levels of UPP exhibited no obvious difference among these groups (data not shown). Intravesical pressure threshold for inducing urethral relaxation was significantly higher in the DM group compared with the control group. However, the threshold was significantly decreased in the DM + GSPE group compared with the DM group $(\mathrm{P}<0.05$; Table II). Furthermore, the UPP nadir during large amplitude bladder contractions was significantly increased in the DM group compared with the control group. In the DM + GSPE group, the UPP nadir was significantly decreased compared with the DM group. These results indicated that DM induces elevation of urethral pressure and dysfunction of urethral relaxation, which subsequently resulted in urination disorder. Whereas, GSPE ameliorated DM-induced dysfunction in urethral relaxation (Fig. 2).
GSPE attenuates oxidative stress in diabetic urethra. The activities of GSH-Px and SOD in urethra tissue were significantly decreased in the DM group compared with the control group ( $\mathrm{P}<0.05$; Fig. 3A and $\mathrm{B})$. However, GSPE administration significantly increased the GSH-Px and SOD activities compared with the DM group ( $\mathrm{P}<0.05$; Fig. $3 \mathrm{~A}$ and $\mathrm{B})$. The level of MDA in the urethra was significantly increased in the $\mathrm{DM}$ group compared with the control group ( $\mathrm{P}<0.05$; Fig. $3 \mathrm{C})$. However, the level of MDA was decreased significantly following the administration of GSPE (P<0.05; Fig. 3C).

GSPE attenuates DM-induced dysfunction in urethral relaxation via the NO-cGMP signaling pathway. To investigate how GSPE attenuated DM-induced dysfunction in urethral relaxation, its role in the NO-cGMP signaling pathway was evaluated. Western blotting was performed to evaluate the expression of nNOS protein in each group. The results indicated a significant decrease of nNOS expression in the DM group compared with the control group $(\mathrm{P}<0.05$; Fig. $4 \mathrm{~A}$ and $\mathrm{B})$. Compared with the DM group, a significant increase in nNOS expression was observed in the DM + GSPE group $(\mathrm{P}<0.05$; Fig. 4A and B). However, no significant difference in nNOS expression was observed in the control group compared with the DM + GSPE group. These results indicated that the protective effects of GSPE in DM-induced urethral dysfunction are associated with the upregulation of nNOS in vivo.

To analyze $\mathrm{ONOO}^{-}$formation in vivo, western blotting and immunohistochemistry were performed to determine the expression of 3-nitrotyrosine in the urethra. Western blotting revealed that the expression of 3-nitrotyrosine in the urethra was significantly increased in the DM group compared with the control $(\mathrm{P}<0.05)$, and significantly decreased in the $\mathrm{DM}+\mathrm{GSPE}$ group compared with the DM group $(\mathrm{P}<0.05$; Fig. 4C and D). The immunohistochemistry results indicated that the immunoreactivity of 3-nitrotyrosine was low in the control group (Fig. 4E). A significant increase in 


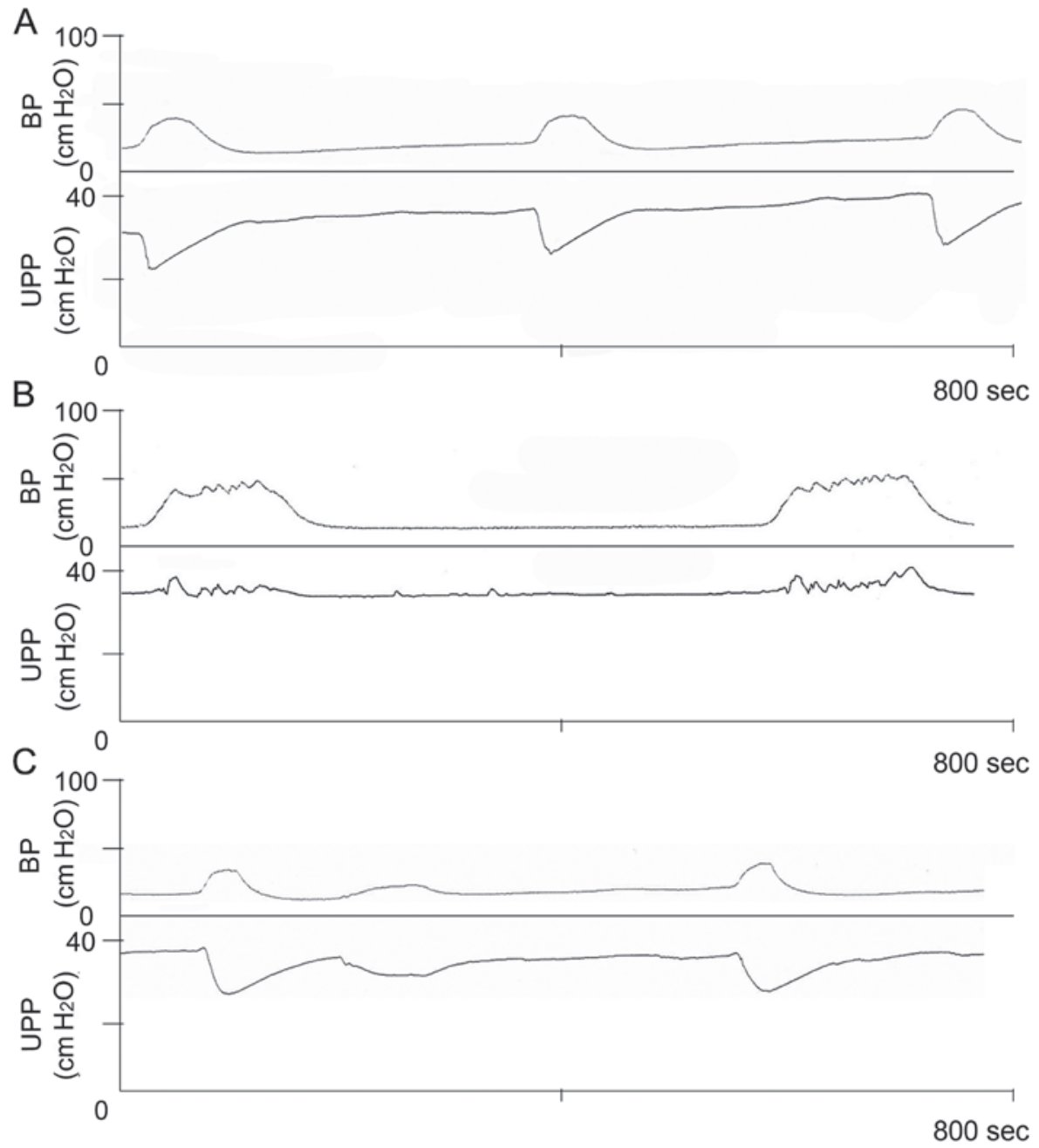

Figure 2. Representative traces of simultaneous recordings of isovolumetric BP and UPP in (A) control rats, (B) diabetic rats and (C) GSPE-treated diabetic rats. BP, bladder pressure; UPP, urethral perfusion pressure; GSPE, grape seed proanthocyanidin extract.
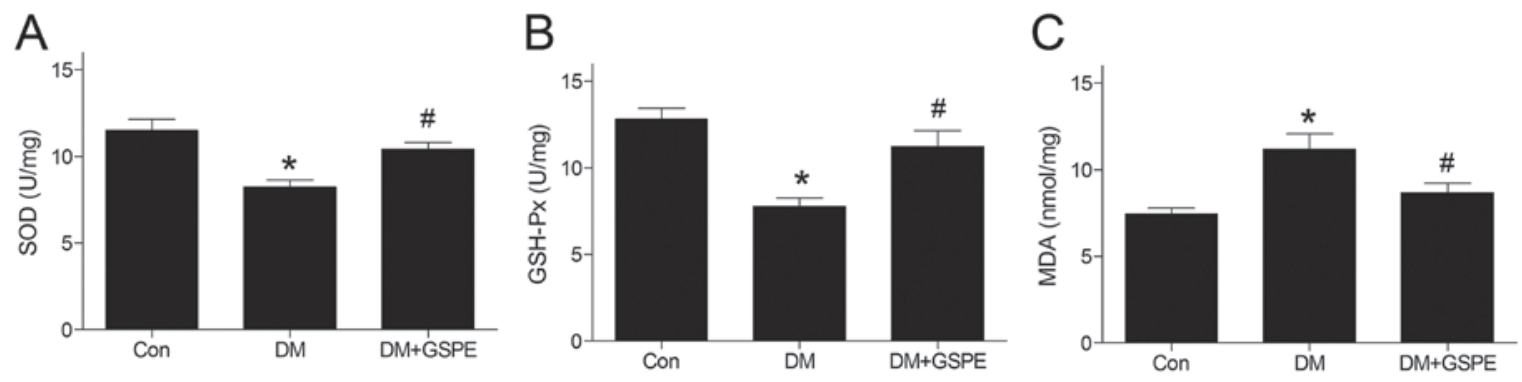

Figure 3. Effect of GSPE on oxidative stress in rat urethra. Oxidative stress was indicated by levels of (A) SOD, (B) GSH-Px and (C) MDA in rat urethra tissue. ${ }^{\text {*P }}<0.05$ vs. control group; ${ }^{~} \mathrm{P}<0.05$ vs. DM group. SOD, superoxide dismutase; GSH-Px, glutathione peroxidase; MDA, malondialdehyde; Con, control; DM, diabetes mellitus; GSPE, grape seed proanthocyanidin extract.

immunoreactivity of 3-nitrotyrosine was observed in the DM group compared with the control group ( $\mathrm{P}<0.05$; Fig. 4E), particularly in the smooth muscle region. In the DM + GSPE group, a significant decrease in the immunoreactivity of 3-nitrotyrosine was observed compared with the DM group $(\mathrm{P}<0.05$; Fig. 4E). These results suggested that GSPE may attenuate the formation of $\mathrm{ONOO}^{-}$in vivo.

For the concentration of $\mathrm{NO}_{\mathrm{x}} \mathrm{N}, \mathrm{DM}$ induced a significant decrease in $\mathrm{NO}_{\mathrm{x}}$ in vivo compared with the control group
$(\mathrm{P}<0.05$; Fig. 5A). However, after administration of GSPE, $\mathrm{NO}_{\mathrm{x}}$ was significantly increased compared with the DM group $(\mathrm{P}<0.05$; Fig. 5A). This suggested that GSPE may upregulate the generation of $\mathrm{NO}_{\mathrm{x}}$ in vivo.

In the NO-cGMP signaling pathway, NO serves a key function in the synthesis of cGMP, which is closely associated with urethral relaxation during micturition reflex. In the present study, a significant decrease in cGMP level was observed in the urethra of DM rats compared with the 

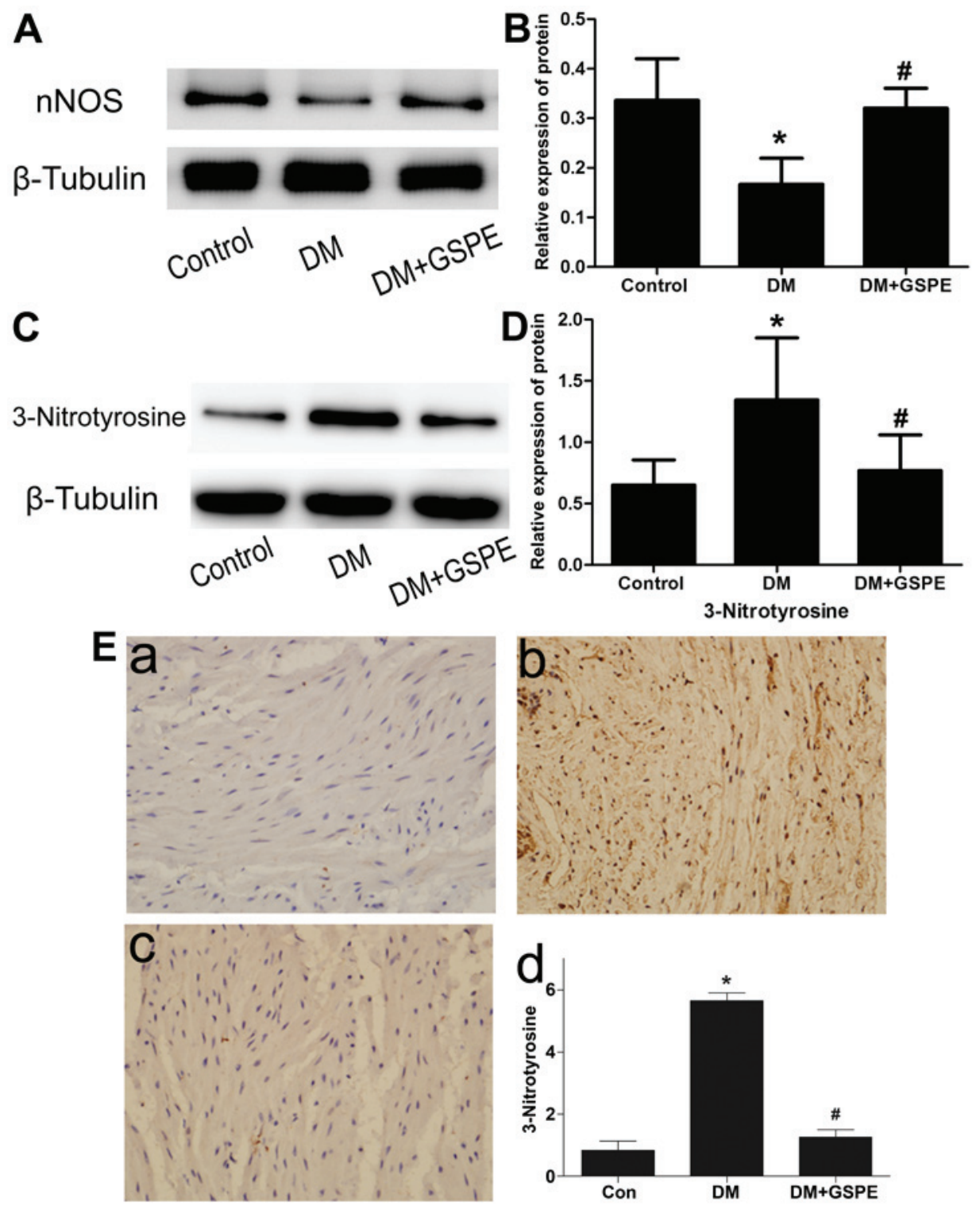

Figure 4. Effect of GSPE on the nitric oxide-cyclic guanosine monophosphate signaling pathway. (A) Western blot analysis and (B) quantification of nNOS expression in urethra tissue. (C) Western blot analysis and (D) quantification of 3-nitrotyrosine expression in urethra tissue. (E) Immunohistochemical staining of 3-nitrotyrosine was performed in tissues from the (a) control, (b) DM group and (c) DM + GSPE group. (d) Quantification of staining. Dark brown color

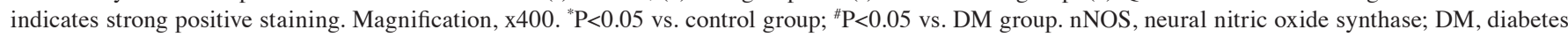
mellitus; GSPE, grape seed proanthocyanidin extract.

control group $(\mathrm{P}<0.05$; Fig. 5B). By contrast, rats treated with GSPE exhibited significantly increased cGMP levels in the urethra compared with the DM group $(\mathrm{P}<0.05$; Fig. 5B). This suggested that GSPE contributes to the upregulation of cGMP in vivo.

Protective effects of GSPE are associated with activation of the Nrf2 pathway. The transcription factor Nrf2 is a vital mediator involved in regulating cellular antioxidative responses. Activation of the Nrf2 pathway leads to neuroprotective effects (20). In the present study, the effects of GSPE on the expression of Nrf2 were investigated. Western blotting (Fig. 6) results demonstrated that the expression of Nrf2 was significantly decreased in the DM group compared with the control $(\mathrm{P}<0.05)$. Nrf2 expression was significantly increased following treatment with GSPE compared with the DM group $(\mathrm{P}<0.05)$. These demonstrated that the protective effects of
GSPE were associated with the activation of the Nrf2 signaling pathway.

\section{Discussion}

DM is a major public health problem worldwide, which causes severe complications and decreases quality of life for sufferers. Although extensive studies have been conducted to identify the potential mechanisms of urethral dysfunction, a common complication in patients with DM $(21,22)$, the exact mechanism is still unclear. In the present study, the antioxidant properties of GSPE were identified to be associated with the repair of urethral dysfunction in DM rats.

Under in vivo conditions, NO is primarily produced by three different NOS isoforms, including nNOS, inducible NOS and endothelial NOS (23). nNOS is specifically expressed in neurons of the nervous system, which results in the generation 
A

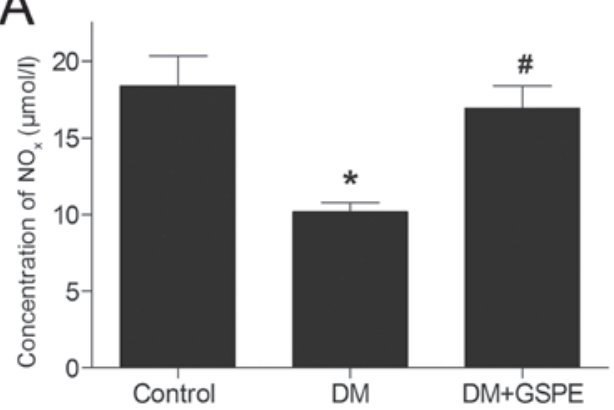

B

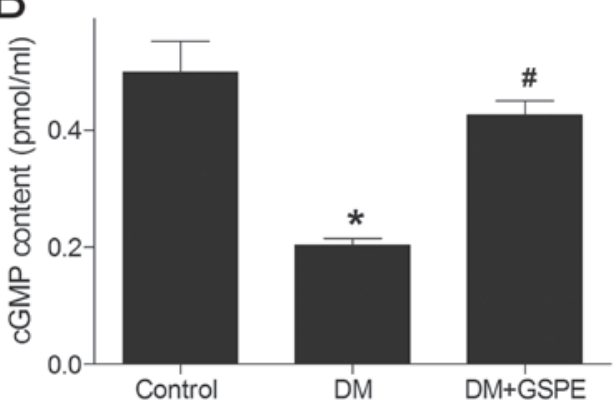

Figure 5. Concentration of $\mathrm{NO}_{\mathrm{x}}$ and cGMP in rat urethra. Levels of $(\mathrm{A}) \mathrm{NO}_{\mathrm{x}}$ and $(\mathrm{B})$ cGMP were measured in urethra tissue. ${ }^{*} \mathrm{P}<0.05$ vs. control group; ${ }^{*} \mathrm{P}<0.05$ vs. DM group. $\mathrm{NO}_{x}$, nitric oxide; cGMP, cyclic guanosine monophosphate; DM, diabetes mellitus; GSPE, grape seed proanthocyanidin extract.

A

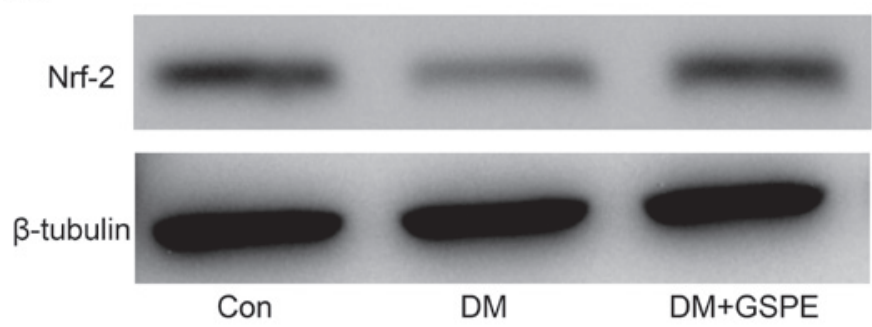

B

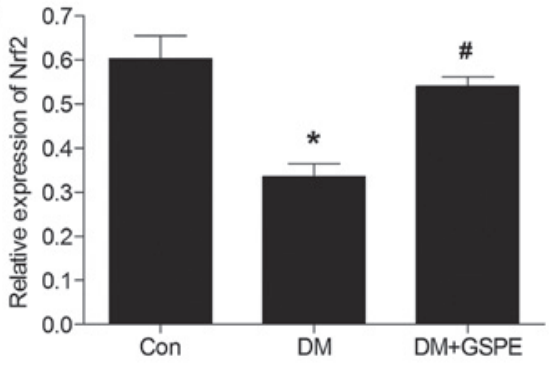

Figure 6. Nrf2 expression in rat urethra tissue. (A) Western blot analysis of Nrf2 expression. (B) Quantification of western blotting results. * $\mathrm{P}<0.05$ vs. control group; ${ }^{\text {P }}<0.05$ vs. DM group. Nrf2, nuclear factor erythroid 2-related factor 2; DM, diabetes mellitus; GSPE, grape seed proanthocyanidin extract.

of nNOS-derived NO. This serves as an atypical neurotransmitter in the peripheral nervous system (10). In addition, nNOS-derived NO exhibits modulatory effects on parasympathetic nerves, which are considered to serve a key function in urethral relaxation during micturition reflex (6). The present results demonstrated that $\mathrm{nNOS}$ production was significantly reduced in the urethra of DM rats. However, in the GSPE group, the production of nNOS was significantly elevated compared with the DM group, indicating that GSPE-mediated protective effects on the urethra may be associated with the elevation of nNOS.

Few studies have been conducted to investigate the NO content in animals with urethral dysfunction. In a previous study, NO production was reported to be increased in the urethra of DM rabbits through the measurement of nicotinamide adenine dinucleotide phosphate diaphorase, a marker of NOS activity (24). However, in the present study, the concentration of NO was determined directly in DM rats, and the results indicated that the NO concentration was significantly decreased in DM rats. Further studies are required in order to explain these inconsistent results.

Previous reports have demonstrated that the cGMP signaling pathway serves a critical function in the regulation of micturition reflex $(25,26)$. It is widely acknowledged that $\mathrm{NO}$ is involved in the mediation of urethral relaxation as it can act on a number of target enzymes and proteins, and is particularly associated with the activation of soluble guanylyl cyclase and the generation of cGMP $(10,27)$. In the DM group of the present study, the expression of cGMP was downregulated. However, in the DM + GSPE group, the expression of cGMP was upregulated compared with the DM group. These

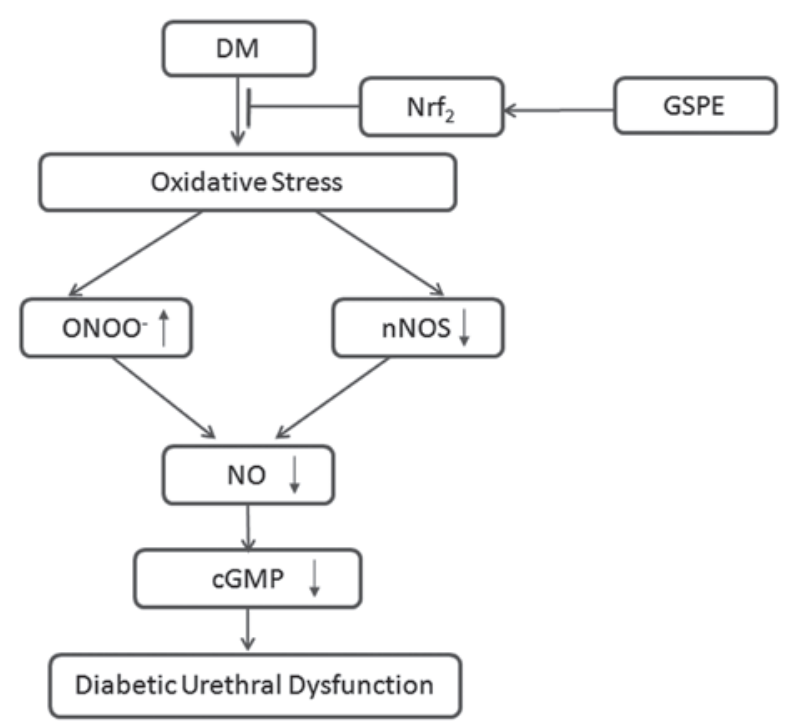

Figure 7. Schematic diagram of the protective mechanism of GSPE against diabetic urethral dysfunction. Arrows indicate activation or induction; bars indicate inhibition or blockade. DM could induce oxidative stress, followed by downregulation of nNOS and upregulation of $\mathrm{ONOO}^{-}$, which synergically result in a decrease of NO formation and bioavailability. Subsequently, this could induce the downregulation of cGMP and cause urethral dysfunction. GSPE could alleviate oxidative stress-induced injuries in DM rats through Nrf2 signaling pathways. Nrf2; nuclear factor erythroid 2-related factor 2; $\mathrm{ONOO}^{-}$, peroxynitrite; nNOS, neural nitric oxide synthase; NO, nitric oxide; cGMP, cyclic guanosine monophosphate; DM, diabetes mellitus; GSPE, grape seed proanthocyanidin extract.

results suggested that GSPE may contribute to the activity of the NO-cGMP signaling pathway. 
$\mathrm{O}_{2}^{-}$can combine with $\mathrm{NO}$ to form $\mathrm{ONOO}^{-}$at a fast rate in $\mathrm{DM}$ (10). The generated $\mathrm{ONOO}^{-}$then inactivates nNOS directly and results in a decrease of the availability of free NO (10). $\mathrm{ONOO}^{-}$may also damage NOS coupling through oxidization of tetrahydrobiopterin to trihydrobiopterin radical, which results in the accumulation of $\mathrm{O}_{2}{ }^{-}$rather than $\mathrm{NO}(10)$. Furthermore, $\mathrm{ONOO}^{-}$may induce toxicity due to its high reactivity and diffusibility (28). Therefore, extensive studies have been performed to reduce oxidative injury through preventing the generation of ONOO (29). In the present study, the effects of GSPE on oxidative stress were investigated in diabetic urethropathy. The results indicated that GSPE could protect urethral function by scavenging free oxygen radicals and enhancing the activities of GSH-Px and SOD. Interestingly, no notable effects were observed on weight loss, hyperglycemia or polyuria in rats with administration of GSPE. On this basis, it can be speculated that GSPE may exert protective effects on the urethra rather than pancreatic beta cells.

The Nrf2 signaling pathway is known to exert antioxidative effects on numerous cell types (30). Food polyphenols could activate the Nrf2 signal pathway (31). In the present study, the results indicated that administration of GSPE could enhance the activity of the Nrf2 pathway in DM rats. In addition, changes in the activity of SOD and GSH-Px corresponded with changes in the Nrf2 pathway. Thus, the current findings suggested that the protective effects of GSPE on the urethra of DM rats may be associated with activation of the Nrf2 signaling pathway.

In summary, the present study demonstrated that GSPE exerts protective effects against diabetic urethral dysfunction in rats. A possible mechanism for this effect is proposed in Fig. 7. DM could induce oxidative stress, followed by downregulation of nNOS and upregulation of $\mathrm{ONOO}^{-}$, which synergically results in a decrease of NO formation and bioavailability. Subsequently, this could induce the downregulation of cGMP and cause urethral dysfunction. GSPE could alleviate oxidative stress-induced injuries in DM rats through Nrf2 signaling pathways.

There are certain limitations of the present study. GSPE is a compound rather than a single monomer. Although the results indicate that GSPE exerts a protective effect on urethra function by modulating the NO-cGMP signaling pathway, the exact active component in GSPE could not be confirmed. Future studies will focus on identifying the active component in GSPE, in order to provide a basis for the research and development of drugs for alleviating urethral dysfunction.

In conclusion, urethral relaxation during reflex bladder contractions is impaired in DM rats. The relaxation may be disturbed by oxidative stress, which would attenuate nNOS and NO levels, finally resulting in a decrease of cGMP formation. GSPE could reduce oxidative stress and protect urethra function by preserving nNOS and NO levels, and contribute to the restoration of urethral relaxation. GSPE treatment may attenuate DM-induced oxidative damage by activating Nrf2.

\section{Acknowledgements}

The present study was supported by the National Natural Science Foundation of China (grant nos. 81470987 and 81170702), the Science and Technology Development Project of Shandong Province (grant no. 2014GSF118054) and the
Medical and Health Science and Technology Development Project of Shandong Province (grant no. 2016WS0019)

\section{References}

1. Yonekubo S, Tatemichi S, Maruyama K and Kobayashi M: Alpha1A-adrenoceptor antagonist improves underactive bladder associated with diabetic cystopathy via bladder blood flow in rats. BMC Urol 17: 64, 2017.

2. Dong X, Song Q, Zhu J, Zhao J, Liu Q, Zhang T, Long Z, Li J, Wu C, Wang Q, et al: Interaction of Caveolin-3 and $\mathrm{HCN}$ is involved in the pathogenesis of diabetic cystopathy. Sci Rep 6: 24844, 2016.

3. Sasaki K, Chanceller MB, Phelan MW, Yokoyama T, Fraser MO, Seki S, Kubo K, Kumon H, Groat WC and Yoshimura N: Diabetic cystopathy correlates with a long-term decrease in nerve growth factor levels in the bladder and lumbosacral dorsal root Ganglia. J Urol 168: 1259-1264, 2002.

4. Yang Z, Dolber PC and Fraser MO: Diabetic urethropathy compounds the effects of diabetic cystopathy. J Uorl 178: 2213-2219, 2007.

5. Bennett BC, Kruse MN, Roppolo JR, Flood HD, Fraser M and de Groat WC: Neural control of urethral outlet activity in vivo: Role of nitric oxide. J Urol 153: 2004-2009, 1995.

6. Persson K, Alm P, Uvelius B and Andersson KE: Nitrergic and cholinergic innervation of the rat lower urinary tract after pelvic ganglionectomy. Am J Physiol 274: R389-R397, 1998.

7. Warner TD, Mitchell JA, Sheng H and Murad F: Effects of cyclic GMP on smooth muscle relaxation. Adv Pharmacol 26: 171-194, 1994.

8. Beshay E and Carrier S: Oxidative stress plays a role in diabetes-induced bladder dysfunction in a rat model. Urology 64: 1062-1067, 2004.

9. Szaleczky E, Prechl J, Feher J and Somogyi A: Alternation in enzymatic antioxidant defence in diabetes mellitus-a rational approach. Postgrad Med J 75: 879, 1999.

10. Förstermann U and Sessa WC: Nitric oxide synthases: Regulation and function. Eur Heart J 33: 829-837, 837a-837d, 2012.

11. Ding Y, Dai X, Jiang Y, Zhang Z, Bao L, Li Y, Zhang F, Ma X, Cai X, Jing L, et al: Grape seed proanthocyanidin extracts alleviate oxidative stress and ER stress in skeletal muscle of low-dose streptozotocin- and high-carbohydrate/high-fat diet-induced diabetic rats. Mol Nutri Food Res 57: 365-369, 2013.

12. Mansouri E, Panahi M, Ghaffari MA and Ghorbani A: Effects of grape seed proanthocyanidin extract on oxidative stress induced by diabetes in rat kidney. Iran Biomed J 15: 100-106, 2011.

13. Casanova E, Baselga-Escudero L, Ribas-Latre A, Cedó L, Arola-Arnal A, Pinent M, Bladé C, Arola L and Salvadó MJ: Chronic intake of proanthocyanidins and docosahexaenoic acid improves skeletal muscle oxidative capacity in diet-obese rats. J Nutri Biochem 25: 1003-1010, 2014.

14. Peng N, Clark JT, Prasain J, Kim H, White CR and Wyss JM: Antihypertensive and cognitive effects of grape polyphenols in estrogen-depleted, female, spontaneously hypertensive rats. Am J Physiol Regul Integr Comp Physiol 289: R771-R775, 2005.

15. Whitman SA, Long M, Wondrak GT, Zheng H and Zhang DD: Nrf2 modulates contractile and metabolic properties of skeletal muscle in streptozotocin-induced diabetic atrophy. Exp Cell Res 319: 2673-2683, 2013.

16. Zhang W, Zhu YH, Zhou D, Wu Q, Song D, Dicksved J and Wang JF: Oral administration of a select mixture of bacillus probiotics affects the gut Microbiota and goblet cell function following Escherichia coli challenge in newly weaned pigs of genotype muc4 that are supposed To Be Enterotoxigenic E. coli F4ab/ac receptor negative. Appl Environ Microbiol 83: pii:e02747-16, 2017.

17. Jung SY, Fraser MO, Ozawa H, Yokoyama O, Yoshiyama M, De Groat WC and Chancellor MB: Urethral afferent nerve activity affects the micturition reflex; implication for the relationship between stress incontinence and detrusor instability. J Urol 162: 204-212, 1999.

18. Zik B, Altunbas K, Tutuncu S, Ozden O, Ozquden Akkoc CG, Peker S and Sevimli A: Effects of capsaicin on nitric oxide synthase isoforms in prepubertal rat ovary. Biotech Histochem 87 : 218-225, 2012.

19. Wang D, Li Y, Hou G, Wang P, Zhang J, Laudon V and Shi B: Pygeum africanum: Effect on oxidative stress in early diabetes-induced bladder. Int Urol Nephrol 42: 401-408, 2010 . 
20. Zhang M, An C, Gao Y, Leak RK, Chen J and Zhang F: Emerging roles of Nrf 2 and phase II antioxidant enzymes in neuroprotection. Proq Neurobiol 100: 30-47, 2013

21. Torimoto K, Hirao Y, Matsuyoshi H, De Groat WC, Chancellor MB and Yoshimura N: Alpha1-adrenergic mechanism in diabetic urethral dysfunction in rats. J Urol 173: 1027-1032, 2005.

22. Masuda H, Tsujii T, Okuno T, Kihara K, Goto M and Azuma H: Localization and role of nitric oxide synthase and endogenous nitric oxide synthase inhibitors in the rabbit lower urinary tract. J Urol 167: 2235-2240, 2002

23. Li H, Jamal J, Plaza C, Pineda SH, Chreifi G, Jing Q, Cinelli MA, Silverman RB and Poulos TL: Structures of human constitutive nitric oxide synthases. Acta Crystallogr D Biol Crystallogr 70 2667-2674, 2014.

24. Mumtaz FH, Sullivan ME, Thompson CS, Dashwood MR, Naseem KM, Bruckdorfer KR, Mikhailidis DP and Morgan RJ: Alterations in the nitric oxide synthase binding sites and non-adrenergic, non-cholinergic mediated smooth muscle relaxation in the diabetic rabbit bladder outlet: Possible relevance to the pathogenesis of diabetic cystopathy. J Urol 162: 558-566, 1999 .
25. Warner T, Mitchell JA, Sheng H and Murad F: Effects of cyclic GMP on smooth muscle relaxation. Adv Pharmacol 26: 171-194, 1994.

26. Feng Z, Wei RB, Hong Q, Cui SY and Chen XM: Grape seed extract enhances eNOS expression and NO production through regulating calcium-mediated AKT phosphorylation in H2O2-treated endothelium. Cell Biol Int 34: 1055-1061, 2010.

27. Caremel R, Oger-Roussel S, Behr-Roussel D, Grise P and Giuliano FA: Nitric oxide/cyclic guanosine monophosphate signalling mediates an inhibitory action on sensory pathways of the micturition reflex in the rat. Eur Urol 58: 616-625, 2010.

28. Marla SS, Lee J and Groves JT: Peroxynitrite rapidly permeates phospholipid membranes. Proc Natl Acad Sci USA 94: 14243-14248, 1997.

29. Szabó C: The pathophysiological role of peroxynitrite in shock, inflammation, and ischemia-reperfusion injury. Shock 6: 79-88, 1996.

30. Kobayashi M and Yamamoto M: Molecular mechanisms activating the Nrf2-Keap1 pathway of antioxidant gene regulation. Antioxid Redox Signal 7: 385-394, 2005.

31. Niture SK, Khatri R and Jaiswal AK: Regulation of Nrf2-an update. Free Radic Biol Med 66: 36-44, 2014. 